Research Paper

\title{
DJ-1 Is Upregulated in Oral Squamous Cell Carcinoma and Promotes Oral Cancer Cell Proliferation and Invasion
}

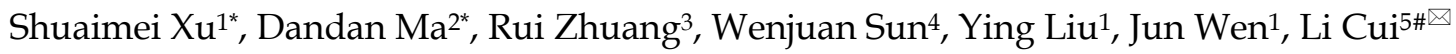 \\ 1. Department of Endodontics, Guangdong Provincial Stomatological Hospital, Guangzhou, China \\ 2. Department of Dentistry, Nanfang Hospital, Guangzhou, China \\ 3. Department of Oral Implantology, School of Stomatology, Capital Medical University, Beijing, China \\ 4. Department of Stomatology, The Third Affiliated Hospital, Sun Yat-sen University, Guangzhou, China \\ 5. Department of Dentistry, Maoming People's Hospital, Maoming, China \\ *These authors contributed equally to this work \\ \#Current address: School of Dentistry, University of California, Los Angeles, California, Unite states of America \\ $\triangle$ Corresponding author: Dr. Li Cui. Tel: 020-84403983; Fax: 020-84403983; Email: zsucllj@ucla.edu
}

(C) Ivyspring International Publisher. Reproduction is permitted for personal, noncommercial use, provided that the article is in whole, unmodified, and properly cited. See http://ivyspring.com/terms for terms and conditions.

Received: 2015.11.26; Accepted: 2016.03.13; Published: 2016.05.24

\begin{abstract}
Background: The development of oral squamous cell carcinoma (OSCC) is a multistep process that involves in both genetic alterations and epigenetic modifications. DJ-1, a negative regulator of tumor suppressor PTEN, functions as an oncogene in many types of cancers. However, its role in OSCC is poorly known.

Methods: Immunohistochemical staining and Western blotting were performed to evaluate the expression level of DJ-1 in oral leukoplakia (OLK) and OSCC tissues respectively. Then lentiviral mediated DJ-1 shRNA was constructed and used to infect the OSCC cell lines (Tca8113 and CAL-27). MTT, cell counting, and Matrigel invasion assay were utilized to examine the effects of DJ-1 down-regulation on proliferation and invasion capacity of oral cancer cells.

Results: The immunoreactivity and expression level of $D J-1$ protein was significantly increased in OLK and OSCC tissues compared with the controls. Lentiviral-delivered shRNA targeting DJ-1 could effectively knock down DJ-1 at mRNA and protein level $(P<0.01)$. The proliferative and invasion ability of OSCC cell lines was significantly suppressed following $D J-1$ inhibition $(P<0.01)$.

Conclusions: Our study indicated that $\mathrm{DJ}-1$ is over-expressed in both oral precancer and cancer tissues and shRNA inhibition of DJ-1 expression led to decreased proliferation and invasion capability of oral cancer cells. These findings suggest that DJ-1 might be actively involved in the development of OSCC. Future studies will investigate the potential of $\mathrm{DJ}-1$ as a biomarker for early detection of OSCC.
\end{abstract}

Key words: DJ-1; Invasion; Oral leukoplakia; Oral squamous cell carcinoma; Proliferation

\section{Introduction}

Head and neck cancer is the sixth most common type of cancer worldwide and oral squamous cell carcinoma (OSCC) represents up to $90 \%$ of all oral malignancies $[1,2]$. OSCC is a multifactorial disease brought on by a combination of genetic alterations, environmental risk factors as well as viral infection.
The current treatments options for OSCC include traditional surgery, radiotherapy, chemotherapy and targeted therapy; and resection is the preferred approach [3]. Although great therapeutic advances have been made in the past three decades, the 5-year survival rate for OSCC has not improved significantly 
and remains at approximately 50\% [4]. The major reasons accounting for the poor prognosis of OSCC are that the malignant disease can be asymptomatic initially and most cases are diagnosed at advanced stage. Thus, understanding the molecular basis of OSCC development, which might help detect this disease at an early stage or even at the precancerous stage, is an effective and practical strategy for improving the clinical outcome.

DJ-1 (PARK7) encodes a protein comprised of 189-amino-acid that is ubiquitously expressed in most mammalian tissues [5]. It was first reported as an oncogene that transformed mouse NIH3T3 cells in combination with activated ras [6], and then identified as a gene linked to Parkinson's disease [7]. This important protein participates in many key cellular processes including, but not limited to, cell proliferation, differentiation, transcriptional regulation, oxidative stress protection, mitochondrial function maintenance, inflammation and metabolic regulation [8-12]. Deregulation of DJ-1 has been implicated in the pathogenesis of many human diseases such as Parkinson's disease, amyotrophic lateral sclerosis, infertility, and cancer [13-16].

Alteration in expression of DJ-1 is associated with tumorigenesis in several types of cancers. In primary breast cancer, the expression level of DJ-1 was inversely associated with PTEN staining and positively correlated with PKB/Akt hyperphosphorylation, indicating that DJ-1 might be the driving force behind cancer development [17]. Morelli et al showed that the serum level of DJ-1 was up-regulated in patients with endometrial cancer compared with healthy controls. In addition, its expression level was associated with tumor grade [18]. The results suggested that detecting the serum DJ-1 expression level might be beneficial to early diagnosis as well as treatment options. The expression level of serum DJ-1 was also reported to be significantly increased in pancreatic cancer patients and DJ-1 played critical roles in regulation of tumor chemoresistance; indicating it might be a potential therapeutic target [19].

Although DJ-1 seems to be a crucial regulator responsible for the initiation and development of cancer, its role in OSCC is poorly documented. Thus, the purpose of the present study was to ascertain the expression level of DJ-1 in oral leukoplakia (OLK) and OSCC; as well as whether DJ-1 modulated the biological functions of OSCC cell lines including proliferation and invasion.

\section{Materials and Methods}

\section{Tissue samples and Immunohistochemistry}

The study was approved by the Ethic Committee of Guangdong Provincial Stomatological Hospital. All tissue specimens were obtained from Department of Oral Pathology, Guangdong Provincial Stomatological Hospital and written informed consent was obtained from all of patients for the use of their tissues. The study specimens comprised of 68 archival cases, of which 15 cases were healthy controls, 17 cases were of OLK and 36 cases of OSCC. For immunohistochemistry analyses, formalin-fixed paraffin-embedded sections were deparaffinized by sequential washing with xylene, 100\% ethanol, 95\% ethanol, $80 \%$ ethanol and PBS. The sections were incubated with $0.3 \% \mathrm{H}_{2} \mathrm{O}_{2}$ in methanol for $5 \mathrm{~min}$ to quench the endogenous peroxidase activity. The slides were blocked in PBS with 5\% BSA for $30 \mathrm{~min}$ and then incubated overnight with a 1:100 dilution of anti-DJ-1 primary antibody (Abcam, MA, USA) at 4 ${ }^{\circ} \mathrm{C}$. After sections were rinsed with PBS, they were incubated with horseradish peroxidase (HRP)-conjugated goat anti-rabbit IgG (1:500, Life Technologies, Carlsbad, CA, USA) for $2 \mathrm{~h}$ at room temperature.

\section{Cell line and cell culture}

The Tca8113 OSCC cell line was purchased from Guangdong Medical Laboratory Animal Center (Guangzhou, China), and the CAL-27 cell line was obtained from XiangYa Central Experiment Laboratory (Changshan, China). Both cell lines were grown in Dulbecco's modified Eagle's medium (Invitrogen, Carlsbad, CA, USA) supplemented with $10 \%$ fetal calf serum (Invitrogen), 100 units/liter penicillin, and $10 \mathrm{mg} /$ liter streptomycin. Cultures were maintained at $37^{\circ} \mathrm{C}$ in an atmosphere containing $5 \% \mathrm{CO}_{2}$.

\section{Western blotting}

Cells were washed twice with ice-cold PBS and lysed with RIPA buffer (Beyotime Biotech, Shanghai, China). Aliquots of cell lysates containing $25 \mu \mathrm{g}$ protein were separated on SDS-polyacrylamide gels and transferred to PVDF membranes at $110 \mathrm{~V}$ for $1.5 \mathrm{~h}$. The membranes were blocked in TBST buffer containing $5 \%$ nonfat milk, and incubated with rabbit antibody against human DJ-1 at a dilution of $1: 200$ (Abcam) overnight, followed by HRP linked goat anti-rabbit IgG (1:5000; Sigma). Bands were visualized using the ECL kit (Beyotime Biotech).

\section{Real-time PCR}

Total RNA was extracted from OSCC cell lines 
using TRIzol (Takara, Dalian, China) following manufacturer's protocol. PrimeScript ${ }^{\mathrm{TM}} \mathrm{RT}$ reagent Kit with gDNA Eraser (Takara) was used to synthesize the first-strand cDNA. The cDNA was then amplified with SYBR Premix DimerEraserTM (Takara) on an ABI 7500 real-time PCR system (Applied Biosystems, CA, USA). Human glyceraldehyde-3phosphate dehydrogenase (hGAPDH) was used as an endogenous control and the $2^{-\Delta \Delta} \mathrm{Ct}$ method was employed to calculate relative expression levels of mRNA. PCR reactions were conducted in triplicate and data were representative of three separate experiments. The primers used for the real-time PCR assays were as follows: DJ-1: Forward: 5'-TGGCTAAAGGAGCAGAGGAA-3', Reverse: 5'ATGACCACATCACGGCTACA-3'; GAPDH: Forward: 5'-TGTTCGTCATGGGTGTGAAC-3', Reverse: 5'-ATGGCATGGACTGTGGTCAT-3'.

\section{Lentivirus infection}

The oligonucleotide sequence used for shRNA targeting DJ-1 was designed as previously described [9]. The negative control was a scrambled sequence with no homology to human genes. The oligonucleotides were first cloned into the GV118-GFP vector (GeneChem, Shanghai, China), then the recombinant lentiviral vectors combined with packaging vectors were co-transfected into $293 \mathrm{~T}$ cells. Lentivirus-containing supernatants were collected at $72 \mathrm{~h}$ after transfection and purified by ultracentrifugation. Lentiviral titers were determined by real-time PCR and OSCC cell lines were infected with lentiviruses at a multiplicity of infection (MOI) of 20.

\section{MTT assay}

After $24 \mathrm{~h}$ of serum starvation, the lentivirus infected OSCC cell lines were seeded into a 96-well plate at a density of 4000 cells/well in $100 \mu \mathrm{L}$ medium for the MTT assay. At each indicated time point, $20 \mu \mathrm{L}$ of MTT (Sigma) dissolved in PBS at $5 \mathrm{mg} / \mathrm{ml}$ was added to each culture well. Following by incubation for $4 \mathrm{~h}$ at $37^{\circ} \mathrm{C}$, the supernatant was then discarded and the precipitate dissolved in $200 \mu \mathrm{l}$ of dimethyl sulfoxide (DMSO, Sigma). The absorbance of each well was measured using a microculture plate reader (Tecan, Mannedorf, Switzerland) at $490 \mathrm{~nm}$. The assay was repeated in triplicate.

\section{Cell counting}

OSCC cells were digested with $0.25 \%$ trypsin to prepare single-cell suspension, followed by counting the cells with a hemocytometer. Then the cells were plated into 12-well plates at a density of $1 \times 10^{5}$ cells per well. At the indicated time points, cell counts were performed in triplicates using a hemocytometer with
$0.2 \%$ trypan blue exclusion to identify viable cells.

\section{Invasion assay}

The invasion assays were performed with matrigel coated transwells ( $8 \mu \mathrm{m}$ pore size; Corning, NY, USA). Following $24 \mathrm{~h}$ serum starvation, cells were harvested and resuspended in DMEM containing $0.1 \%$ FBS, and $2 \times 10^{5}$ cells were then added to upper chamber of transwell inserts. DMEM containing 10\% FBS was used as chemoattractant in the lower chamber. After $48 \mathrm{~h}$, cells that had migrated through the membrane were fixed in methanol and stained with $0.1 \%$ crystal violet. The invaded cells in four random fields were counted and expressed as the average number of cells per field under light microscopy (Eclipse TE2000, Nikon, Tokyo, Japan).

\section{Statistical analysis}

The data were expressed as the mean \pm standard deviation, and analyzed by the independent samples t-test using SPSS v21.0 software (SPSS Inc, Chicago, IL, USA). $P$ values $<0.05$ were regarded to be statistically significant.

\section{Results}

\section{Expression of DJ-1 in OLK and OSCC}

IHC and Western blotting were performed to evaluate the expression level of DJ-1 in clinical samples. The positive staining of epithelium cells was identified as yellow-brown granules. In normal epithelium, positive staining was almost absent and detected only in the most active parabasal and basal layers (Fig. 1A-1B). In OLK tissues, the immunoreactivity intensity of epithelial peg was strong and the positive staining had the tendency to spread to other layers of the epithelial (Fig. 1C-1D). In OSCC tissues, the area of positive staining was observed in the whole epithelial and staining density was stronger than OLK tissue (Fig. 1E-1F). DJ-1 positive cells were found in tumor nest (Fig. 1G-1H).

Western blotting showed that DJ-1 was increased significantly in both OLK and OSCC tissues compared with the matched adjacent tissues (MAT) (Fig. 1I), which was consistent with IHC results and further confirmed that DJ-1 was overexpressed in OLK and OSCC tissues.

\section{DJ-1 shRNA lentiviruses inhibit DJ-1 mRNA and protein expression}

Both Tca8113 cells (Fig. 2A-2B) and CAL-27 cells (Fig. 2C-2D) were successfully infected with GFP lentiviruses that expressed either DJ-1 shRNA or scrambled shRNA. Real-time PCR and Western blotting results showed that both messenger RNA and protein expression levels were significantly lower in 

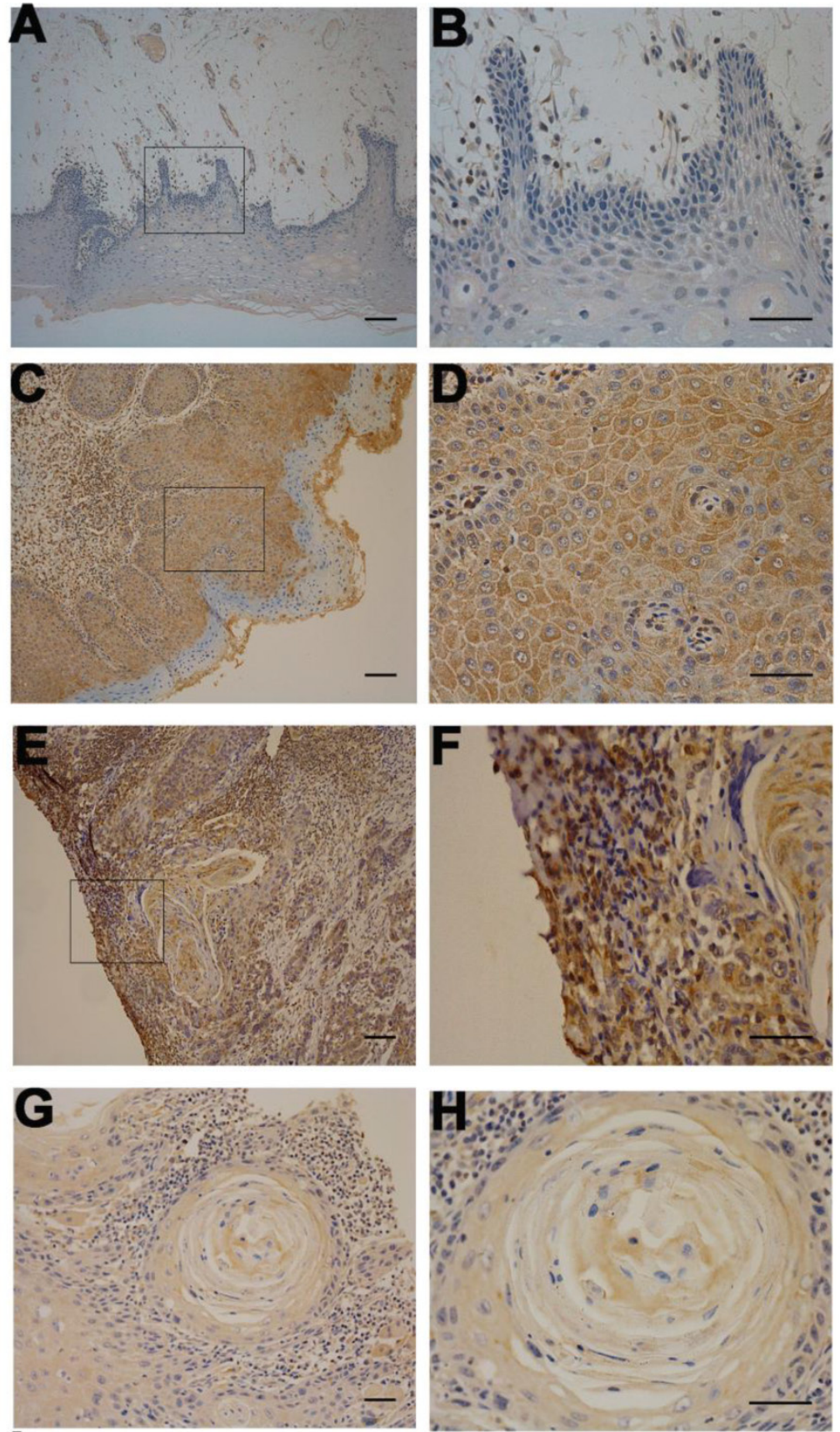

1

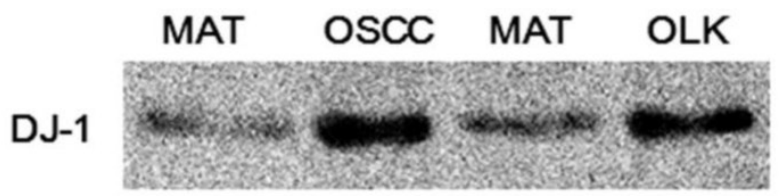

$\beta$-actin

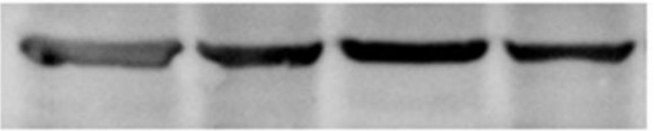

Figure 1. Expression of DJ-1 in OLK and OSCC tissues. (A-B) DJ-1 was rarely detected in the epithelial of normal tissue. (C-D) Positive staining was found mostly in the basal part of the epithelial in the OLK tissue. (E-F)Widespread expression of DJ-1 in the whole epithelial of infiltrated OSCC tissue. (G-H) DJ-1 positive cells were also detected in the cancer nest. (I) The Western blot results showed that the expression level of DJ-1 protein was increased in OLK and OSCC tissues compared with matched adjacent tissues (MAT). the DJ-1 inhibition cells compared with the controls (Fig. 2E-2G, ${ }^{* *} P<0.01,{ }^{* * *} P$ $<0.001$ ).

\section{Down-regulation of DJ-1 inhibited proliferation capacity of OSCC cell lines}

To assess the role of DJ-1 in the proliferation of OSCC, MTT and cell counting assay were performed using oral cancer cells (Tca8113 cells and CAL-27 cells) infected with lentiviruses expressing specific shRNA against DJ-1 or scrambled shRNA at different time points $(24,48,72$ and 96h). The results showed that the number of oral cancer cells in the DJ-1 suppression group was significantly lower in comparison with the negative control group at specific time points $\left({ }^{*} P<0.05,{ }^{* *} P\right.$ $<0.01$ ) (Fig. 3A-3D).

\section{Down-regulation of DJ-1 inhibited invasion capability of OSCC cell lines}

To investigate whether DJ-1 involves in regulation invasion capability of OSCC cell lines, matrigel invasion assay was used to evaluate the motility of oral cancer cells after DJ-1 suppression. Compared with scrambled shRNA control cells, DJ-1 knockdown Tca8113 cells showed about $75 \%$ reduction in the number of cells crossing the membrane $(P<0.01)$ (Fig. 4A, $4 \mathrm{~B}$ and $4 \mathrm{E}$ ). Similarly, the average number of CAL-27 cells (negative control) that migrated through the membrane was $67 \pm$ $9 /$ per field, whereas only $21 \pm 8$ /per field CAL-27 cells that subjected to DJ-1 inhibition reached the lower chamber $(P<0.01)$ (Fig. 4C, 4D and 4F). The results indicated that DJ-1 may play a crucial role in the regulation of oral cancer cells invasion.

\section{Discussion}

In this study, our results showed that the expression level of DJ-1 was significantly increased in both OLK and OSCC tissues. In addition, downregulation of DJ-1 inhibited the proliferation and invasion capacity of OSCC cell lines; suggesting DJ-1 may act as an oncogene during the tumorigenesis process of OSCC. 

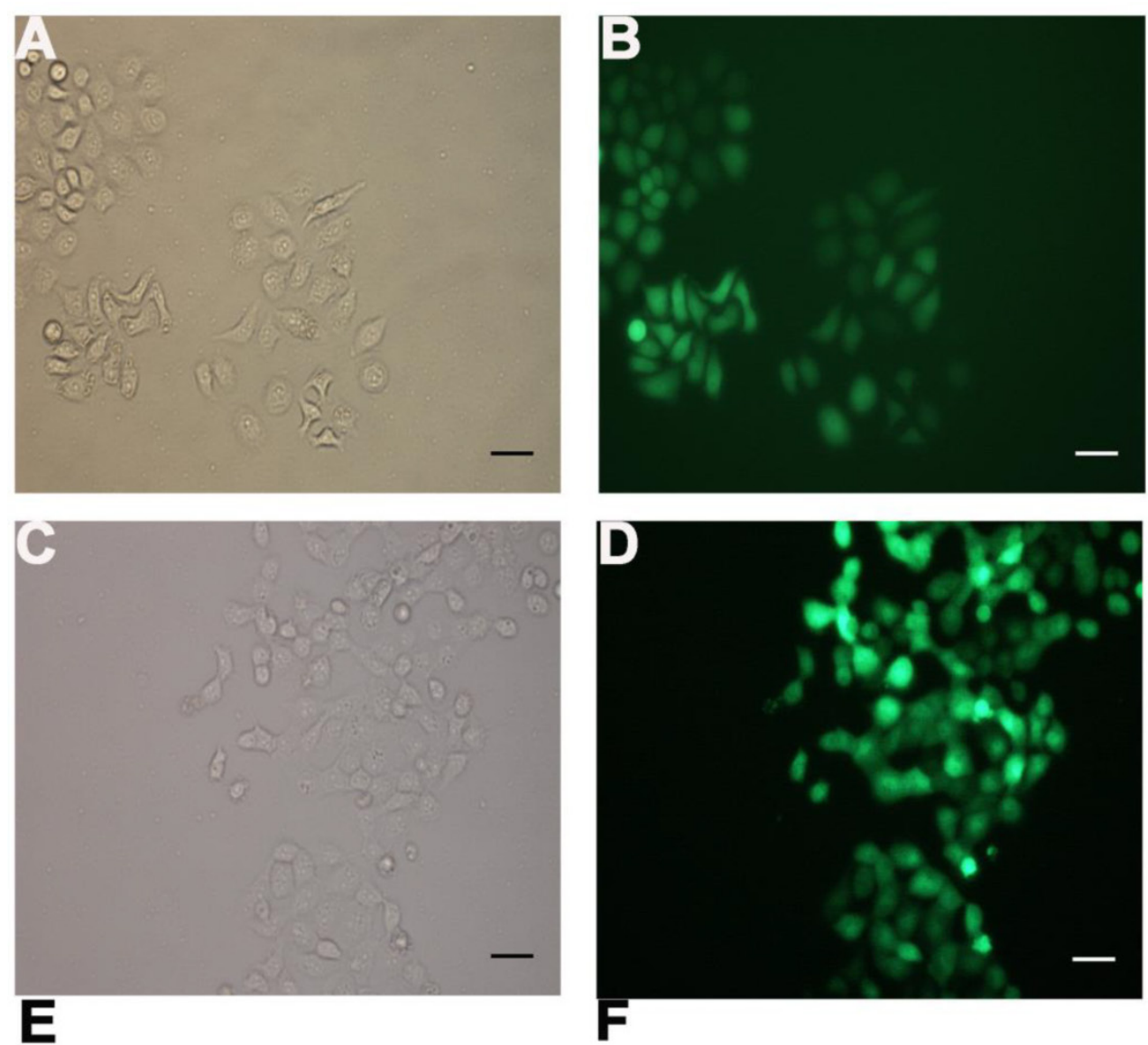

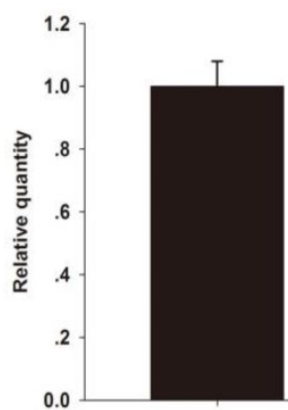

NC

Tca8113 DJ-1 mRNA

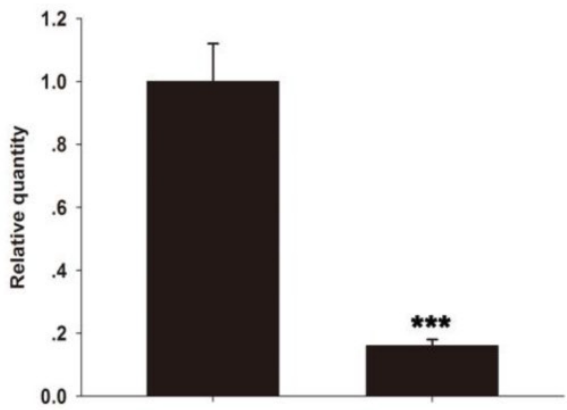

NC

G

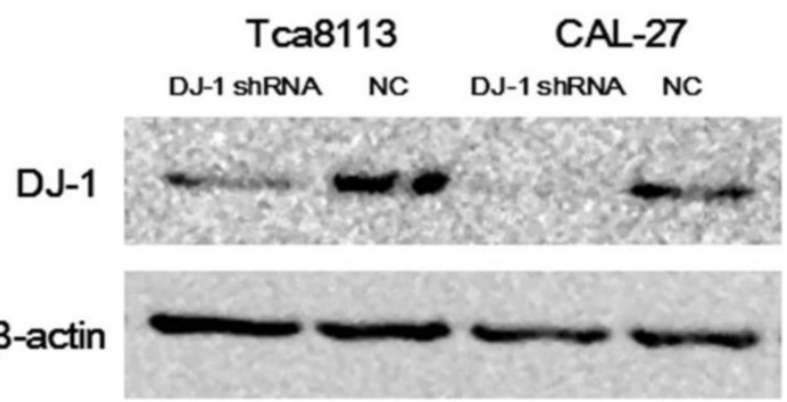

Figure 2. Infection of oral cancer cells with lentiviruses expressing DJ-1-specific shRNA or control (scrambled) shRNA. GFP fluorescence was observed in the Tca8113 cells (A-B) and CAL-27 cells (C-D) respectively, allowing infection efficiency to be monitored. (E-G) Real-time PCR and Western blotting showed that the expression levels of DJ-1 mRNA and protein were significantly down-regulated after lentiviruses infections $(* * P<0.01, * * * P<0.001)$. Each experiment was repeated three times. All values are expressed as the mean \pm standard deviation 



Figure 3. DJ-1 downregulation inhibited proliferation of oral cancer cells. (A-B) The MTT result showed that the OD values of the Tca8113 cells infected with lentiviruses that expressed DJ-1 shRNA were significantly lower compared with the negative controls at all-time points except the first day $(* * P<0.01)$. Similar findings was also observed using the cell counting assay $\left({ }^{*} P<0.05\right.$, $\left.{ }^{*} * P<0.01\right)$. (C-D) As regards to CAL-27 cells, MTT showed that the OD values of $C A L-27$ cells in the $D J-1$ knock-down group were remarkably reduced in comparison with the cells in the negative control group at 48,72 and $96 \mathrm{~h}$ after seeding $(* P<0.05, * * P<0.01)$. Statistical significant results were found at 72 and $96 \mathrm{~h}$ in the cell counting assay $(* P<0.05, * * P<0.01)$. Each experiment was repeated three times. All values are expressed as the mean \pm standard deviation.

Oral squamous carcinogenesis is a multistep process that involves in activation of proto- oncogenes and inactivation of tumor suppressor genes [20]. However, the underlying molecular mechanism remains largely unknown. A large proportion of OSCC tumors develop from oral precancerous lesions such as OLK, oral submucous fibrosis, oral erythroplakia and actinic cheilitis [21]. OLK is the most commonly occurring precancerous lesion, thus it is a good clinical model for understanding the tumorigenesis of oral cancer. Both the immunoreactivity and the expression level of DJ-1 was upregulated in OLK tissues in compared with the controls. To the best our knowledge, this is the first report on the association between OLK and DJ-1 expression level. In addition, the overexpression of DJ-1 in OLK indicated that DJ-1 might actively participate in regulation of OSCC at the precancerous stage. What is more, this finding has potential clinical significance, it is possible that evaluating the expression level of tissue DJ-1 help early detection of
OSCC. This was consistent with previous literature that elevated serum level of DJ-1 was found to be associated with multiple risk factors of choroidal nevi transformation including symptoms, nevi thickness; basal diameter as well as the presence of acoustic hollowness; indicating DJ-1 might help the precancerous lesion evolve into melanoma [22].

Similarly, overexpression of DJ-1 was observed in OSCC tissues. In most cases, the immunoreactivity intensity of DJ-1 was stronger in OSCC tissues compared with in OLK tissues; suggesting that DJ-1expression level might be closely correlated the clinical stage and severity of OSCC. Wang et al compared the differentially expressed proteins between OSCC tissues and healthy tissues using the proteomic method. DJ-1 expression was found to be significantly upregulated in OSCC [23]. Upregulation of DJ-1 has also been reported in other types of cancers including head and neck cancer. DJ-1 was overexpressed in supraglottic squamous cell carcinoma (SSCC). Moreover, higher DJ-1 expression 
was linked to worse nodal status and shorter 5-year overall survival; indicating that DJ-1 might be a promising prognostic indicator for SSCC [24]. Davidson et al reported that higher expression level of DJ-1 mRNA was frequently observed in advanced-stage ovarian carcinoma regardless of the anatomical sites and associated with worse prognosis [25]. However, the role of DJ-1 in regulation of
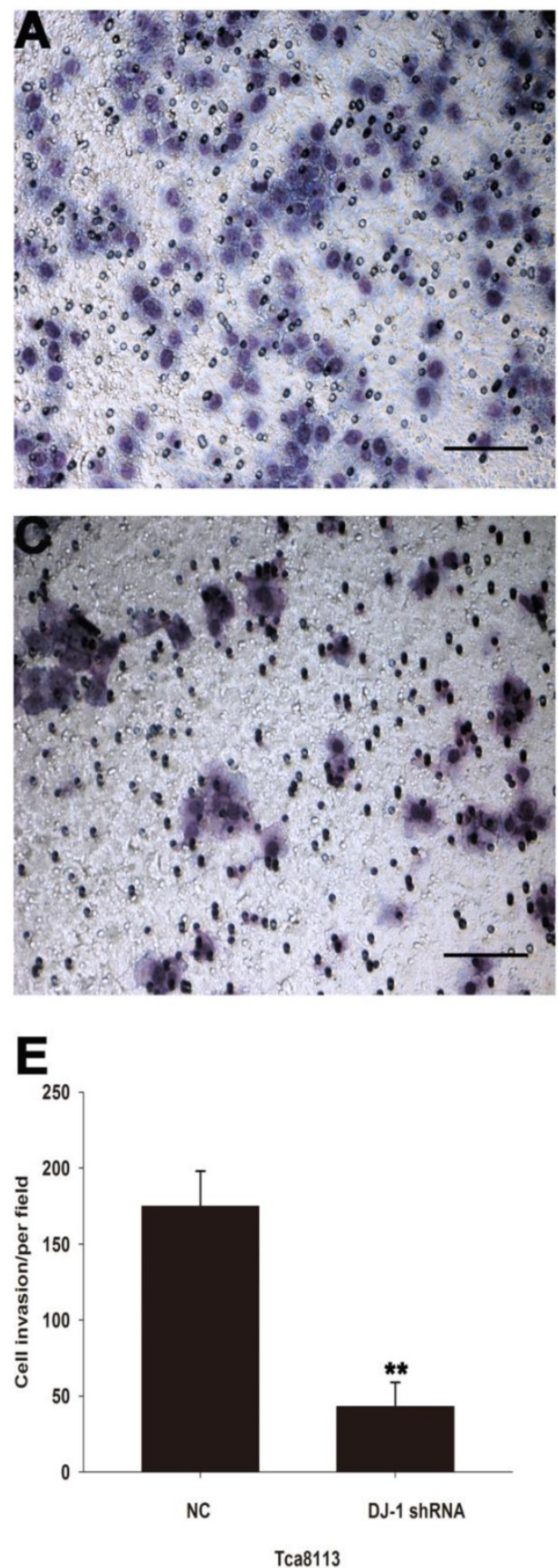

tumorigenesis was complex and not consistent in all types of cancers investigated so far. The astrocytoma patients with lower nuclear expression level of DJ-1 had significantly shorter overall survival, suggesting that DJ-1 probably played a tumor suppressive role in astrocytoma [26]. Possible explanations are that the function of DJ-1 might be cell type dependent and it is associated with tumor microenvironment.


$\mathbf{F}$

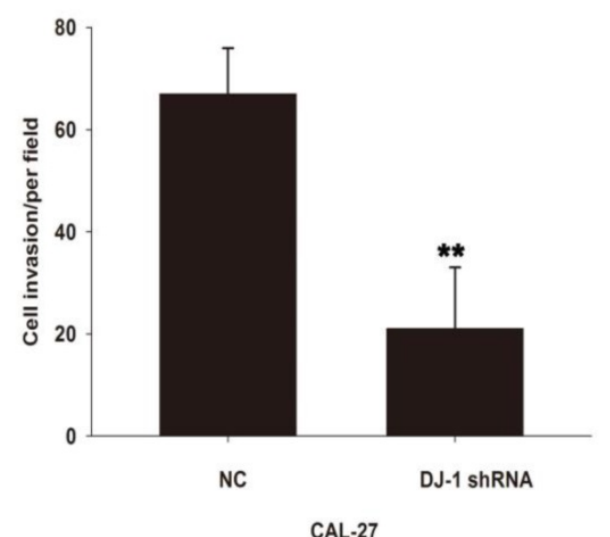

Figure 4. $D$ J-1 suppression inhibited the invasion capacity of OSCC cells. (ABE) The number of $D$ J-1 knock-down Tca8113 cells that migrated through the membrane was significant less than the negative controls $(* * P<0.01$ ). (CDF) Similarly, the number of DJ-1 suppressed CAL-27 cells in the lower chamber was significant less than the cells in the negative control group $(* * P<0.01)$. Each experiment was repeated in triplicate. All values are expressed as the mean \pm standard deviation. 
Our results revealed that the lentiviruses we used could infect the OSCC cell lines with high efficiency and effectively know-down the expression of DJ-1 at both mRNA and protein level, which laid a solid foundation for investigating the role of DJ-1 in OSCC. Currently lentiviral vectors are among the most efficient gene transfer tools. They have several obvious advantages such as incorporating into genomic DNA with high efficiency, maintaining the transgene expression for long periods of time and lacking of viral genes transfection [27]. Suppression of DJ-1 could strongly inhibit proliferation and invasion capacity of OSCC in vitro; which indicated that DJ-1 overexpression in OSCC might promote the tumorigenesis process and further corroborated the oncogenic role of DJ-1 in OSCC. PTEN is a known tumor suppressor and DJ-1 was shown to be a negative regulator of PTEN. Thus it is possible that inhibiting the expression level of DJ-1 might upregulate the activity of PTEN, which might then exert its anti-tumor functions via targeting the downstream signaling pathways. Vasseur and his colleagues showed that DJ-1 and p53 are tightly correlated. p53 could prevent DJ-1 protein accumulation, whereas loss of p53 led to DJ-1 stabilization and enhancement[28]; indicating DJ-1 might be a key player in the development of cancer.

One limitation of our study is the sample size of clinical specimens was relatively small and the observation time was short. Thus it was difficult for us to examine whether there were direct associations between tissue DJ-1 expression level and OLK/OSCC clinical parameters as well as prognosis. Further research is necessary to provide more evidence to elucidate the clinical significance of DJ-1 in OLK and OSCC. Body fluids have become a useful diagnostic tool for many human diseases. It is not only less invasive than tissue biopsy, but also enable the clinicians to monitor the diseases in real-time. Thus detecting the expression level of DJ-1 in body fluids such as saliva, serum and plasma of patients with OLK or OSCC is also a future direction. Although we found that down-regulation of DJ-1 could inhibit the oncogenic behaviors of OSCC cell lines, investigations that address the precise molecular mechanism of DJ-1 in OSCC and its role in vivo are required.

Taken together, our data demonstrated that DJ-1 was overexpressed in OLK and OSCC, and it involved in mediating cancer cell proliferation and aggressiveness in OSCC; indicating DJ-1 might have substantial clinical impact and the potential to be a candidate biomarker of OSCC.

\section{Acknowledgments}

This study was supported by the Medical Scientific Research Foundation of Guangdong Province (Grant no. B2014411, L.C.), National Natural Science Foundation of China (Grant no. 81400495, D.D.M) and Guangdong Natural Science Foundation (2015 A03030101, D.D.M).

\section{Competing Interests}

The authors have declared that no competing interest exists.

\section{References}

1. Ferlay J, Shin HR, Bray F, et al. Estimates of worldwide burden of cancer in 2008: GLOBOCAN 2008. Int J Cancer. 2010; 127:2893-917.

2. Choi S, Myers JN. Molecular pathogenesis of oral squamous cell carcinoma: implications for therapy. J Dent Res. 2008; 87:14-32.

3. Feller L, Lemmer J. Oral squamous cell carcinoma: epidemiology, clinical presentation and treatment. J Cancer Ther. 2012; 3:263-8.

4. Fuller CD, Wang SI, Thomas CR Jr, et al. Conditional survival in head and neck squamous cell carcinoma: results from the SEER dataset 1973-1998. Cancer. 2007; 109:1331-43.

5. Bandopadhyay R, Kingsbury AE, Cookson MR, et al. The expression of DJ-1 (PARK7) in normal human CNS and idiopathic Parkinson's disease. Brain. 2004; 127:420-30.

6. Nagakubo D, Taira T, Kitaura $\mathrm{H}$, et al. DJ-1, a novel oncogene which transforms mouse NIH3T3 cells in cooperation with ras. Biochem Biophys Res Commun. 1997; 231:509-13

7. Huai $Q$, Sun $Y$, Wang $H$, et al. Crystal structure of DJ-1/RS and implication on familial Parkinson's disease. FEBS Lett. 2003; 549:171-5.

8. Won $\mathrm{KJ}$, Jung $\mathrm{SH}$, Lee $\mathrm{CK}$, et al. DJ-1/park7 protects against neointimal formation via the inhibition of vascular smooth muscle cell growth. Cardiovasc Res. 2013; 97:553-61.

9. Kim JM, Shin HI, Cha SS, et al. DJ-1 promotes angiogenesis and osteogenesis by activating FGF receptor-1 signaling. Nat Commun. 2012; 3:1296.

10. Martinat C, Shendelman S, Jonason A, et al. Sensitivity to oxidative stress in DJ-1-deficient dopamine neurons: an ES- derived cell model of primary Parkinsonism. PLoS Biol. 2004; 2:e327.

11. Hayashi T, Ishimori C, Takahashi-Niki K, et al. DJ-1 binds to mitochondrial complex I and maintains its activity. Biochem Biophys Res Commun. 2009; 390:667-72.

12. Kim JM, Jang HJ, Choi SY, et al. DJ-1 contributes to adipogenesis and obesity-induced inflammation. Sci Rep. 2014; 4: 4805.

13. Bonifati V, Rizzu P, van Baren MJ, et al. Mutations in the DJ-1 gene associated with autosomal recessive early-onset parkinsonism. Science. 2003; 299:256-9.

14. Lev N, Ickowicz D, Barhum Y, et al. DJ-1 changes in G93A-SOD1 transgenic mice: implications for oxidative stress in ALS. J Mol Neurosci. 2009; 38:94-102.

15. An CN, Jiang H, Wang $Q$, et al. Down-regulation of DJ-1 protein in the ejaculated spermatozoa from Chinese asthenozoospermia patients. Fertil Steril. 2011; 96:19-23.

16. Cao J, Lou S, Ying M, et al. DJ-1 as a human oncogene and potential therapeutic target. Biochem Pharmacol. 2015; 93:241-50.

17. Kim RH, Peters M, Jang $Y$, et al. DJ-1, a novel regulator of the tumor suppressor PTEN. Cancer Cell. 2005; 7:263-73.

18. Morelli M, Scumaci D, Di Cello A, et al. DJ-1 in endometrial cancer: a possible biomarker to improve differential diagnosis between subtypes. Int J Gynecol Cancer. 2014; 24:649-58.

19. Chen Y, Kang M, Lu W, et al. DJ-1, a novel biomarker and a selected target gene for overcoming chemoresistance in pancreatic cancer. J Cancer Res Clin Oncol. 2012; 138:1463-74.

20. Williams HK. Molecular pathogenesis of oral squamous carcinoma. Mol Pathol. 2000; 53: 165-72.

21. Humayun S, Prasad VR. Expression of p53 protein and ki-67 antigen in oral premalignant lesions andoral squamous cell carcinomas: An immunohistochemical study. Natl J Maxillofac Surg. 2011; 2:38-46

22. Bande MF, Santiago M, Blanco MJ, et al. Serum DJ-1/PARK 7 is a potential biomarker of choroidal nevi transformation. Invest Ophthalmol Vis Sci. 2012; 53:62-7.

23. Wang $\mathrm{Z}$, Jiang $\mathrm{L}$, Huang $\mathrm{C}$, et al. Comparative proteomics approach to screening of potential diagnostic and therapeutic targets for oral squamous cell carcinoma. Mol Cell Proteomics. 2008; 7:1639-50.

24. Zhu XL, Wang ZF, Lei WB, et al. Tumorigenesis role and clinical significance of DJ-1, a negative regulator of PTEN, in supraglottic squamous cell carcinoma. J Exp Clin Cancer Res. 2012; 31:94.

25. Davidson B, Hadar R, Schlossberg A, et al. Expression and clinical role of DJ-1, a negative regulator of PTEN, in ovarian carcinoma. Hum Pathol. 2008; 39:87-95. 
26. Miyajima $\mathrm{Y}$, Sato $\mathrm{Y}$, Oka $\mathrm{H}$, et al. Prognostic significance of nuclear DJ-1 expression in astrocytoma. Anticancer Res. 2010; 30:265-9.

27. Park F. Lentiviral vectors: are they the future of animal transgenesis? Physiol Genomics. 2007; 31:159-73.

28. Vasseur S, Afzal S, Tomasini R, et al. Consequences of DJ-1 upregulation following p53 loss and cell transformation. Oncogene. 2012; 31:664-70. 